\title{
Impact of routine varicella immunization on varicella ambulatory visits in a Tertiary Hospital in Athens, Greece
}

\author{
Papaevangelou Vassiliki ${ }^{*}$, Douros Konstantinos ${ }^{1}$, Mavrikou Myrsini ${ }^{2}$, Iliopoulou Anna ${ }^{3}$ and Tsolia Maria ${ }^{4}$ \\ ${ }^{1}$ Third Department of Pediatrics, National and Kapodistrian University of Athens Medical School, General University Hospital “ATTIKON”, Athens, Greece \\ ${ }^{2}$ First Department of Pediatrics, 'P \& A. Kyriakou' Childrens' Hospital, Athens, Greece \\ ${ }^{3}$ Second Department of Pediatrics, 'P \& A. Kyriakou' Childrens' Hospital, Athens, Greece \\ ${ }^{4}$ Second Department of Pediatrics, National and Kapodistrian University of Athens Medical School, 'P \& A. Kyriakou' Childrens' Hospital, Athens, Greece
}

\begin{abstract}
The impact of routine varicella immunization on the incidence of varicella infection among children in Greece has not been evaluated. The rates of varicella infection ambulatory visits between 1.1.1998 to 31.12.2014 was studied. The incidence rates were calculated based on the total number of emergency department attendances during each year of the study period and interrupted time series analysis was performed. Furthermore, changes among Greek and minority children were compared. Introduction of varicella vaccination in 2004 had a significant effect on varicella and an annual decease of varicella ambulatory visits at a rate of -7.30 (CI= -13.30 , $-1.29, \mathrm{p}=0.02$ ) was observed. Ambulatory visits decreased significantly more among Greek children when compared to minority children. In conclusion, following the introduction of routine varicella immunization, the rate of varicella ambulatory visits has decreased notably among children in Greece. However, further decrease among underserved minority groups should be achieved.
\end{abstract}

\section{Abbreviations: VZV: Varicella Zoster Virus; HZ: Herpes Zoster}

\section{Introduction}

Varicella is a highly contagious disease caused by primary infection with the varicella zoster virus (VZV), characterized by a benign self-limiting clinical course [1]. However, common complications (including bacterial super-infection of skin lesions, pneumonia, otitis media, febrile seizures, encephalitis and ataxia) may occur among healthy children [1]. Morbidity rates are elevated among young infants, adults, and immunocompromised patients [1]. Moreover, following primary infection, VZV becomes latent in the dorsal root ganglia and may reactivate later in life causing herpes zoster (HZ), a localized painful rash often associated with severe morbidity. As the disease burden of VZV is notable, routine immunization against varicella has been implemented in several countries and resulted in a significant decrease of varicella infection rates [2]. In particular, in the USA where routine vaccination has been implemented since 1995, significant decreases in the incidence rate and attributable mortality of varicella, as well as in varicella-related hospitalizations, have been documented [2-4]. In Greece, routine varicella immunization was initiated during 2004 and 2 years later was introduced in the Hellenic National Immunization Program and therefore fully compensated. In 2009 reimbursement for the booster injection was endorsed. National immunization rates for the initial and booster immunizations approximate $90 \%$ and $75 \%$, respectively [5]. Uncomplicated varicella is not a notifiable disease, therefore data demonstrating the impact of routine immunization are lacking. The herein described retrospective observational study aimed to examine the impact of universal varicella vaccination on varicella ambulatory visits in the emergency outpatient pediatric clinic department of a large tertiary hospital.

\section{Materials and methods}

The study was conducted at the "P. \& A. Kyriakou" Children's Hospital in Athens, Greece. The study site constitutes one of the two largest referral tertiary centers located in Greece and serves approximately 650,000 children aged $0-14$ years. The hospital's outpatient Emergency Department is functional every second day, including weekends, and thus carries the main burden of pediatric care in Athens. After the study protocol was approved by the hospital Ethics Review Board, the emergency attendances records of the Pediatric Department for the period January 1, 1998 through December 31, 2014 were reviewed. The annual total number of patients examined in the Pediatric Emergency Rooms, as well as the number of patients who presented with signs and symptoms indicative of varicella were recorded. Data concerning date (month-year) of hospital visit, age and ethnicity were recorded only for VZV patients. Diagnosis was confirmed by an attending pediatrician. To estimate whether the introduction of varicella vaccination resulted in a shift of varicella incidence and trend, we performed interrupted time series analysis using an ordinary leastsquares regression-based approach which allowed for autocorrelation adjustment [6].

Moreover, the study period was divided in three periods based

Correspondence to: Papaevangelou Vassiliki, Third Department of Pediatrics, National and Kapodistrian University of Athens Medical School, General University Hospital "ATTIKON", Athens, Greece, Tel: +30-2105832228; Fax: +30-2105832229; E-mail:vpapaev@med.uoa.gr

Key words: varicella; ambulatory visits; vaccine; minority groups

Received: August 17, 2016; Accepted: September 24, 2016; Published: September 27, 2016 
on varicella vaccine implementation: (1) Pre-licensure period (19982003) (2) Interim period (2004 - 2006) and (3) Post-licensure period (2007-2014). The interim period was excluded from comparisons since vaccination was not reimbursed and therefore varicella vaccine uptake was lower. Age distribution, and ethnicity of varicella patients were compared by the Rao-Scott Chi-Square test. The pre-licensure period was applied as the basis of comparison for analysis. $P$ values $<0.05$ were considered the criterion of significance. SAS version 9.1 was applied for all analyses.

\section{Results}

During the study period, 819,488 patients visited the ER at the study site, amongst which, $4,357(0,53 \%)$ were due to varicella (mean age: 5.86 years; range $0.5-15$ years) (Figure 1 ). The overall mean rate of ambulatory visits due to varicella was 50 cases per 10,000 visits. As shown in Table 1, the annual number of ambulatory visits significantly decreased over the study period. Interrupted time series analysis showed that introduction of varicella vaccination in 2004 had a significant effect of on varicella incidence. Our model predicted a significant decrease in varicella incidence in the first year (2004) of the intervention $(-55.36,95 \% \mathrm{CI}=-95.58,-15.13, \mathrm{p}<0.0001)$, followed by a significant decrease in the annual trend of incidence (relative to the preintervention trend) of -14.98 decrease of incidence per year $(95 \% \mathrm{CI}=$ $-27.66,-2.29, p=0.024)$. It also predicts that after the introduction of vaccination, varicella incidence deceased annually at a rate of -7.30 $(95 \% \mathrm{CI}=-13.30,-1.29, \mathrm{p}=0.020)$.

When pre- and post-licensure periods were compared, the rate of varicella ambulatory visits was significantly decreased $(\mathrm{p}<0,001)$ among Greek children (1844 versus 213) when compared to minority children (1095 versus 352). Minority children included immigrant and Roma children. Moreover, the mean age of children presenting with varicella did not significantly change (5.9 versus 5.68 years) between the two aforementioned periods.

\section{Discussion}

This retrospective study demonstrates a noteworthy decrease in the varicella ambulatory visit rate in a large tertiary pediatric hospital in Athens post implementation of varicella immunization [4]. Greece is one of the few European countries where routine varicella vaccination has been implemented [http://vaccine-schedule.ecdc. europa.eu]. Complicated varicella cases are reported. However, since

\section{Ambulatory visits 1998 - 2014}

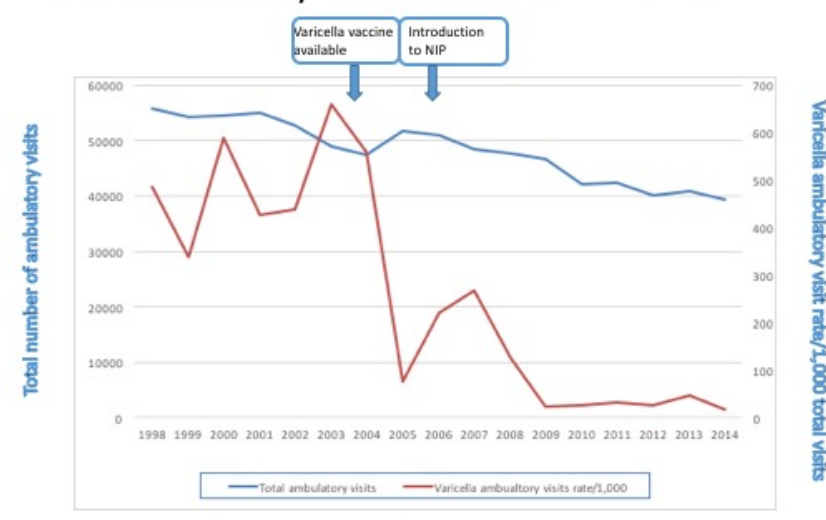

Figure 1. Total number of ambulatory visits in emergency department and varicella ambulatory visit rate over the study period.
Table 1. Annual number and rates of varicella ambulatory visits.

\begin{tabular}{|c|c|c|c|c|}
\hline Year & ED (n)* & $\begin{array}{c}\text { Number of varicella } \\
\text { cases }\end{array}$ & $\begin{array}{c}\text { Varicella rate } \\
\text { (per 10,000) }\end{array}$ & $\mathbf{9 5 \%}$ CI \\
\hline 1998 & 55836 & 485 & 86.86 & $79.30-94.70$ \\
\hline 1999 & 54243 & 339 & 62.49 & $56.03-69.49$ \\
\hline 2000 & 54608 & 590 & 108.04 & $99.54-117.07$ \\
\hline 2001 & 54973 & 428 & 77.85 & $70.67-85.56$ \\
\hline 2002 & 52797 & 438 & 82.95 & $75.39-91.06$ \\
\hline 2003 & 49049 & 659 & 134.35 & $124.35-144.93$ \\
\hline 2004 & 47481 & 559 & 117.73 & $108.22-127.84$ \\
\hline 2005 & 51835 & 75 & 14.46 & $11.38-18.13$ \\
\hline 2006 & 50915 & 219 & 43.01 & $37.51-49.08$ \\
\hline 2007 & 48465 & 268 & 55.29 & $48.89-62.30$ \\
\hline 2008 & 47670 & 125 & 26.22 & $21.83-31.23$ \\
\hline 2009 & 46607 & 23 & 4.93 & $3.12-7.40$ \\
\hline 2010 & 42210 & 26 & 6.15 & $4.02-9.02$ \\
\hline 2011 & 42381 & 33 & 7.78 & $5.36-10.93$ \\
\hline 2012 & 40011 & 26 & 6.49 & $4.25-9.52$ \\
\hline 2013 & 40994 & 46 & 11.22 & $8.21-14.96$ \\
\hline 2014 & 39413 & 18 & 4.56 & $2.70-7.21$ \\
\hline & & & & \\
\hline
\end{tabular}

uncomplicated varicella is not a reportable disease there is considerable under-reporting which impedes the estimation of epidemiology trends of varicella disease. Recent report from CDC also recognizes that continued efforts are needed to accurately estimate varicella burden of disease and vaccination impact [4]. This is the first report from Greece post implementation of universal varicella vaccination. Although a national vaccination coverage study performed in 2012 indicated that vaccine uptake was remarkable, other smaller studies indicate that there might be regional differences in vaccination coverage [7]. Importantly, recent study in the beginning of the economic crisis in Greece has indicated a sustainable vaccination coverage [8]. However, improved surveillance, maintenance of vaccination coverage and more importantly identification and vaccination of susceptible adults at risk is important nowadays with the increased influx of refugees. This is quite important, since increased incidence of varicella outbreaks in refugee camps have been reported (unpublished data).

Importantly, the finding that varicella still prevails among the underserved minority groups is worrisome. This increases the risk of accumulation of susceptible population, mainly older children and adolescents, for whom VZV infection may be more severe. Although the mean age of varicella cases remained stable, it is important to increase surveillance in order to identify possible age shifts over the next years.

The most important limitation of this study is that data presented refer to a single tertiary pediatric center. Therefore, data might not be representative of the general population. Also, data vaccination status of the varicella cases was not consistently reported and therefore the attribution of breakthrough cases could not be ascertained.

In conclusion, the data presented indicate that introduction of universal varicella vaccination of toddlers in Greece has resulted in a significant reduction of disease incidence as depicted by the reduction of varicella ambulatory visit rate. Moreover, there is evidence that minority children might be under-vaccinated. Although no age shift of varicella cases was observed, the study findings call for public health action in order to enhance varicella immunization coverage particularly among minority adolescents. Such public health initiatives would potentially diminish the population at-risk for contracting varicella infection at older ages when the clinical consequences of infections are most severe. 


\section{Acknowledgements}

We would sincerely like to thank all the young doctors that helped in the data collection: Klinaki Eleni, Koutsoubari Ioanna, Chairetaki Vassiliki, Gioka Katerina.

\section{References}

1. Arvin AM (1996) Varicella-zoster virus. Clin Microbiol Rev 9: 361-381. [Crossref]

2. Papaloukas O, Giannouli G, Papaevangelou V2 (2014) Successes and challenges in varicella vaccine. Ther Adv Vaccines 2: 39-55. [Crossref]

3. Baxter R, Ray P, Tran TN, Black S, Shinefield HR, et al. (2013) Long-term effectiveness of varicella vaccine: a 14-Year, prospective cohort study. Pediatrics 131: e1389-1396. [Crossref]

4. Lopez AS, Zhang J, Marin M (2016) Epidemiology of Varicella During the 2-Dose
Varicella Vaccination Program -United States, 2005-2014. MMWR Morb Mortal Wkly Rep 65: 902-905. [Crossref]

5. Panagiotopoulos T, Georgakopoulou T, Stavrou D, Danis K, Laggas D, et al. (2012) National study of vaccination coverage among children. Athens: National School of Public Health.

6. Linden A (2015) Conducting interrupted time series analysis for single and multiple group comparisons. Stata Journal 15: 480-500.

7. Pavlopoulou ID, Michail KA, Samoli E, Tsiftis G, Tsoumakas K (2013) Immunization coverage and predictive factors for complete and age-appropriate vaccination among preschoolers in Athens, Greece: a cross--sectional study. BMC Public Health 13: 908. [Crossref]

8. Vassiliki P, Ioanna K, Artemis V (2014) Determinants of vaccination coverage and adherence to the Greek national immunization program among infants aged 2-24 months at the beginning of the economic crisis (2009-2011). BMC Public Health 14: 1192. [Crossref]

Copyright: (C2016 Vassiliki P. This is an open-access article distributed under the terms of the Creative Commons Attribution License, which permits unrestricted use, distribution, and reproduction in any medium, provided the original author and source are credited. 